\title{
AUTOUR DU RÉFÉRENT, UNE CONSTRUCTION ORIGINALE DE LA LINGUISTIQUE ANTIQUE TARDIVE
}

\author{
MARC BARATIN \\ Université de Lille 3 (France)
}

\begin{abstract}
RÉSUMÉ: Larticle se propose de reconstituer le système conceptuel dans lequel se développe la notion de persona dans l'Ars grammatica de Priscien. Plutôt qu'une notion centrale, pourtant, on y trouve un ensemble complexe qui, construit sur des critères distincts, permet d'aborder la question de la construction et de l'identification de la référence à plusieurs niveaux: dans le domaine proprement référentiel, c'est-à-dire extra-linguistique, réel ou fictif, avec le suppositum, entité du monde connu du locuteur et/ou de l'allocutaire; dans le cadre sémantique d'une catégorie linguistique déterminée (nom, pronom, verbe "être"), avec la substantia, "être" en tant qu'il est inscrit dans le domaine linguistique; sur un plan pragmatique dans le processus énonciatif, avec la persona, protagoniste jouant un rôle particulier qui le distingue des autres; enfin d'un point de vue syntaxique par rapport au procès lui-même, qu'il réalise ou subit, avec l'agens et le patiens, actant d'un procès exprimé par le verbe. L'une des difficultés du système ainsi mis en place tient, comme souvent dans le vocabulaire technique antique, à la porosité des frontières entre ces notions, qui se chevauchent à l'occasion.
\end{abstract}

MOTS-CLEFS: Priscien; référent; persona; suppositum et hupokeimenon; substantia et qualitas.

La distinction dans les analyses linguistiques entre les plans intra- et extralinguistiques suppose la mise en place des différents concepts nécessaires au passage de l'un à l'autre de ces plans. Du point de vue de l'histoire de la linguistique, l'apparition et l'organisation de ces concepts, et par là la genèse d'une pensée de l'articulation de ces deux plans, est un champ d'autant plus fertile que chaque époque ne met pas l'accent sur les mêmes éléments. A cet égard, le dernier et le plus 
important texte linguistique de l'Antiquité tardive, l'Ars grammatica de Priscien ${ }^{1}$, présente un intérêt historique particulier dans la mesure où ce texte constitue le maillon entre les conceptions développées dans le monde grec et celles des Latins, ainsi qu'entre la linguistique antique et le monde médiéval. Or les appréciations portées par les Modernes sur cet aspect du texte de Priscien soulignent surtout les faiblesses du dispositif mis en place. On a relevé notamment l'ambiguïté de la notion de persona, à la fois être humain et catégorie grammaticale tripartite $\left(1^{\mathrm{re}} / 2^{\mathrm{e}} / 3^{\mathrm{e}}\right.$ pers.):

alors que Priscien, à la suite du grammairien grec Apollonios qui lui sert en l'occurrence de modèle, distingue au moins implicitement les personnes physiques impliquées dans la communication verbale et les trois personnes grammaticales, il faut pourtant reconnaître que (...) les deux acceptions du mot sont souvent confondues² (Amacker 1990: 271).

Cette constatation n'est pas négligeable, mais elle cache peut-être l'essentiel, c'est-à-dire l'organisation même du système conceptuel qui permet et produit la confusion dénoncée par les Modernes. La critique de confusion s'appuie en effet sur une sorte d'a priori: dès lors que les deux plans sont objectivement distincts, et que Priscien lui-même les distingue à l'occasion - fût-ce implicitement -, le fait qu'il lui arrive de les superposer ou de les mêler ne pourrait relever que d'une faiblesse du raisonnement. Et ce type d'interprétation vient conforter l'image de grammairiens antiques incapables de construire des principes explicatifs stables, qui vaillent pour la totalité de l'œuvre où ils sont produits (Amacker 1990: 269).

Du point de vue de l'histoire de la linguistique, une question préalable s'impose néanmoins: la confusion relevée et dénoncée aujourd'hui comme une bévue n'est-elle pas en fait conditionnée par le dispositif d'analyse mis en place dans les textes antiques où on l'identifie?Poser cette question revient à inverser les termes du problème: au lieu de postuler que la grille d'analyse du grammairien ancien est la même que celle des Modernes, mais qu'il l'utilise de façon erronée (pour quelle raison?), il s'agirait de savoir si la grille d'analyse n'est pas elle-même différente de celle des Modernes, de telle façon qu'une distinction qui s'impose pour les uns

1 Actif à Constantinople au début du $6^{\mathrm{e}}$ s., il y enseignait la langue latine à un public hellénophone. Son œuvre majeure, l'Ars grammatica date de 526-527.

2 Avec pour exemple, notamment, GL 2, 423.8 etsq. 
pourrait tout aussi bien être reconnue ou ne pas l'être par les autres. Le premier objectif à atteindre est donc de reconstituer le système conceptuel en question.

Cela étant, avant même d'entrer dans ce système, ou plutôt à titre de préalable pour y entrer, il convient de souligner une forte divergence avec les Modernes dans la façon d'appréhender la question de la référence. La linguistique contemporaine s'est beaucoup interrogée sur la question de la réalité des référents auxquels des expressions linguistiques renvoient: s'agit-il d'entités indépendantes du langage, auxquelles des expressions linguistiques permettent de renvoyer, ou s'agitil en fait de constructions linguistiques?Cette dernière hypothèse, spécifiquement contemporaine, se heurte à l'évidence de l'expérience lorsque le référent est le voisin de palier, l'ordinateur sur lequel on travaille, ou encore le château de Versailles. La première hypothèse en revanche paraît supposer une existence objective des référents, qui seraient des entités du monde réel, indépendantes du langage: la référence serait alors une relation entre la langue et le monde, et établirait un lien entre des expressions linguistiques et des entités du monde réel. Cette conception, volontiers présentée comme classique (au sens de "non contemporaine" ${ }^{\text {") }}$, s'est vue opposer par les Modernes l'obstacle que constituent les entités imaginaires, dénotées par des expressions comme "la licorne", "Pégase", "le Père Noël”, etc. Si l'on admet que ces expressions ont bien un référent, il faut étendre l'accès de la référence à des mondes autres que le seul monde réel et intégrer ces mondes fictifs - mais cela remet en cause le réalisme objectiviste de cette conception de la référence.

Dans des travaux récents (1997 et 1999), Georges Kleiber met ce débat à la base de l'analyse contemporaine de la référence. Il est d'autant plus intéressant de constater que, pour les Anciens, et en l'occurrence pour Priscien, le problème ne se pose pas en ces termes. Les êtres qu'il cite comme référents sont pour une très large part des entités fictives: il les emprunte aux textes littéraires qui constituent le socle culturel qu'il partage avec les lecteurs auxquels il destine ses analyses. Ainsi, dans un

3 Cf. Kleiber (1999: 17): "Il est tout naturel qu'on pense avant tout à une existence objective, c'est-à-dire une existence dans la réalité. Les référents sont des entités du monde réel, indépendantes du langage, auxquelles on renvoie précisément à l'aide des expressions linguistiques. Et la référence apparaît alors comme étant une relation languemonde, qui établit un lien entre une portion ou des segments du monde réel et des expressions linguistiques. (...) La conception classique de la référence s'accompagne donc d'un engagement ontologique en faveur de l'existence dans ce qui constitue notre monde ou la réalité d'êtres et de choses auxquelles on peut référer avec les expressions linguistiques". 
des passages qu'il consacre à ce sujet dans le livre 17 de son Ars, Priscien examine différents aspects de l'identification d'un référent en se fondant sur un épisode de l'Énéide où Anchise aux Enfers montre à Énée les âmes qui bientôt feront l'histoire romaine (GL 3, 123.1-12). L'exemple censé illustrer ici la désignation d'un être situé hors du champ linguistique est ainsi placé aux Enfers, dans le cadre d'un texte littéraire... A l'occasion, Priscien attire lui-même l'attention sur le caractère fictif des situations décrites: "Même en sachant en effet que Virgile est poète et fils de Maro, si jamais nous l'apercevions, à supposer que cela fût possible, nous ne saurions pas que c'est le nom de cette personne-là, à moins qu'on ne nous l'ait désigné en disant: hic est Vergilius [voici Virgile]"4. Assurément, toutes les situations évoquées par Priscien, ou tous les référents, ne sont pas fictifs, mais cette réflexion sur Virgile montre qu'il a pleinement conscience que certains ne relèvent en aucune façon de la réalité. Surtout, Priscien n'établit aucune différence entre les uns et les autres: la question de la réalité ou non des référents lui est indifférente. On pourrait penser que c'est seulement un mécanisme qui l'intéresse: il se placerait dans une perspective objectiviste, en faisant comme si les exemples qu'il cite étaient tous des entités du monde réel. En fait, il paraît plus vraisemblable que si Priscien n'aborde pas ce genre de questions, c'est que pour lui le problème se pose en termes différents.

Ce qui l'intéresse en effet, c'est d'envisager le problème de la référence non pas sous l'angle de la réalité (ou de l'irréalité) de l'entité concernée, mais sous l'angle de la connaissance qui en est manifestée, ou, en d'autres termes, sous l'angle du mécanisme cognitif que l'expression de la référence suppose ou révèle. Ainsi, lorsque Priscien décrit une situation qui implique de renvoyer à un référent, ou lorsqu'il examine une expression linguistique qui comporte ce genre de renvoi, il les aborde à partir de ce que savent, ou ne savent pas, le locuteur et/ou l'allocutaire. Priscien revient là-dessus à plusieurs reprises et de diverses façons, notamment dans le livre 17. Il passe ainsi en revue différents types d'interrogations, pour préciser à quoi correspond chacune du point de vue de ce que connaît le locuteur et de ce qu'il cherche à connaître. En voici quelques exemples, parmi beaucoup d'autres:

quis mouetur? [qui bouge?], quis ambulat?[qui marche?], quis loquitur? [qui parle?]: "on cherche à connaître la substance d'un référent, l'acte lui-même étant patent, c'est-à-dire le mouvement,

4 GL 3, 146.4-6: Quamuis enim sciamus quod poeta sit Virgilius et filius Maronis, cernentes eum, si posset fieri, nesciebamus, eius esse hoc nomen, nisi si qui nobis eum demonstrans dixisset: "hic est Vergilius". Dans la traduction, c'est nous qui soulignons, bien sûr. 
la marche ou la parole, mais la personne qui l'accomplit restant indéterminée" (GL 3, 122.1-4)

quis legit?-Trypho-qualis?-sapiens, doctus [qui lit?- Tryphon - lequel?le sage, le savant]: "on connaît préalablement le "qui", c'est-à-dire qu'on connaît une substance, et on s'interroge sur sa qualité (sc. sur ses propriétés)" (122.21-23) ;

quis est ille Romanus formosus et magnus? [qui est ce Romain beau et grand?]: "la substance et la qualité une fois formulées, ainsi que les autres accidents qui les accompagnent, on pose en plus une question qui concerne ce propre qui est son nom, car je peux, en voyant quelqu'un, connaître sa substance, sa nationalité, sa qualité, sa taille, et m'interroger seulement sur ce qui lui est propre à lui" (123.1-5, et cf. 129.16-17)

qualiter homo uicit? [comment l'homme a-t-il vaincu?]: "on ne pose pas de question sur l'homme, mais sur les modalités de son acte" $(130.13-15)^{8}$;

quid agit? [que fait-il?] ou quid patitur? [que lui arrive-t-il?]: "on cherche à connaître l'acte effectué ou l'acte subi exprimé par un verbe" $(131.19-21)^{9}$.

Le point de départ de ces analyses est toujours de type formel: les différentes questions examinées sont d'abord décrites en termes de parties du discours, nom, pronom ou adverbe, et verbe, éventuellement le verbe "être", mais ce qui est construit sur cette base formelle n'est pas une analyse de type syntaxique au sens classique

5 Substantiam alicuius suppositi quaerentes dicimus "quis mouetur ?", "quis ambulat?", "quis loquitur?", cum manifestus sit actus, id est motus uel ambulatio uel loquella, persona uero agens incerta.

6 Si praenoscitur quis sit, id est si de substantia sciamus et qualitatem quaerimus, tunc supra dictis utendum est, ut puta "quis legit? - Trypho - qualis? - sapiens, doctus".

7 Est tamen et cum substantia et qualitate in ore posita et aliis coaccidentibus additur interrogatio, pertinens ad proprietatem nominis, ut si dicam "quis est ille Romanus formosus et magnus?"; possum enim, uidens aliquem, scire eius et substantiam et gentem et qualitatem et quantitatem, proprietatem uero solam quaerere.

8 Qui uero interrogat "qualiter homo uicit?" de homine non dubitat, sed de modo actus.

9 Verbi actum uel passionem quaerentes "quid agit?" uel "quid patitur?" dicimus. 
du terme, mais pragmatique: ce qui est pris en considération pour chacune de ces questions, c'est le type d'informations auquel elle correspond, et la présentation du type d'informations qu'elle appelle.

Priscien distingue en outre deux niveaux de connaissance, selon qu'elle est "première" ou "seconde". Cette distinction, d'origine aristotélicienne, est empruntée au modèle grec de Priscien, le grammairien Apollonios, et plus précisément à ses analyses de l'article: le grammairien grec se sert de cette opposition binaire pour différencier ce qui relève d'une information nouvelle ("connaissance première") et ce qui est déjà connu ("connaissance seconde") (Apollonios, Syntaxe, 1.71). On en retrouve la trace littérale chez Priscien, qui l'évoque à propos de la langue grecque: "L'article indique un second niveau de connaissance des référents. Si en effet je dis anthrôpos êlthen [un-homme est venu (sans article)], je manifeste un premier niveau de connaissance; mais avec ho anthrôpos êlthen [1'homme est venu], un second niveau"10 (124.14-16). Conformément à ce qu'implique cette analyse de l'article grec, Priscien associe systématiquement les notions de connaissance première et de connaissance seconde avec celles de "deixis" et d' "anaphore": la prima cognitio d'une entité procède d'une perception directe de celle-ci, per demonstrationem (et par effet induit cette deixis fait connaître de surcroît les caractéristiques et propriétés de cette entité "dans la mesure où on peut les apercevoir en regardant" (146.1718)); cependant que la secunda cognitio procède quant à elle relatione, c'est-à-dire par rappel de ce qui est déjà connu ${ }^{11}$.

Cette opposition (prima cognitio / deixis vs secunda cognitio / anaphore) correspond, au moins en première approximation, à la distinction actuelle entre exophore, pointage vers un référent présent dans la situation d'énonciation, et endophore, pointage vers un antécédent textuel. Priscien présente à l'occasion cette opposition comme correspondant à la présence ou à l'absence du référent: "la deixis... expose au regard ce que l'on fait savoir des présents; l'anaphore... renvoie à ce que l'on sait déjà des absents" (144.5-7). Cette distinction de la présence ou de l'absence du référent est cependant sans doute due à un désir de simplification pédagogique, et elle est elle-même nuancée dans d'autres passages du livre 17: pour tenir compte de l'emploi de déictiques alors que le référent n'est pas à proprement parler "présent" (au sens où il serait possible de le montrer dans la situation

10 Articulus secundam notitiam suppositorum demonstrat. Si enim dicam ö $v \theta \rho \omega \pi \circ \varsigma \tilde{\eta} \lambda \theta \varepsilon v$, primam notitiam ostendo; $\sin \alpha ّ \nu \theta \omega \pi \circ \varsigma \bar{\eta} \lambda \theta \varepsilon v$, secundam.

11 Cette distinction est déjà clairement formulée au début du livre 12, consacré au pronom, GL 2, 579.15-27, et cf. dans le livre 17, GL 3, 128.17-19; 139.26-29. 
d'énonciation), Priscien oppose à la deixis "de la vue" une deixis "de l'intellect", lorsque la référence visée par le déictique n'est pas immédiatement visible, ou qu'il s'agit d'une entité abstraite (142.17-24 et 143.1-15); il souligne d'autre part dans le même passage que l'entité à laquelle renvoie l'anaphore peut assurément être absente de la situation d'énonciation, mais peut tout aussi bien y être présente, pourvu qu'elle soit déjà connue (143.15-26).

En fait, ces apparentes hésitations, voire ces flottements dans l'analyse du référent lui-même (est-il présent ou absent quand il y a deixis? est-il ou non absent quand il y a anaphore?), tiennent à ce que, dans le processus référentiel, Priscien s'intéresse prioritairement, sinon exclusivement, à ce que l'énoncé référentiel suppose ou révèle en matière d'informations à propos de l'auteur de cet énoncé. C'est la notion de "connaissance", et à travers elle ce qui relève de l'information déjà acquise, et des compléments éventuels qui y sont apportés, qui occupe une place centrale dans l'analyse de la référence, et c'est cette réflexion sur le niveau ou le type de connaissance du locuteur, ou de l'allocutaire, qui fédère l'ensemble de ces analyses des expressions linguistiques correspondant aux moyens de construire une référence ou de l'identifier, et qui en constitue le facteur commun.

Limportance de cette réflexion est telle qu'à l'occasion Priscien compare différentes formulations avec pour seul critère "ce que sait" ou "ne sait pas" l'allocutaire:

Vergilius in VII: est mihi nata..., pro possideo natam, sed magis datiuo quasi ad ignorantes utimur, ut in supra dicto uersu; nam ad scientes, esse natam, nomen autem proprium ignorantes, dixisset mea uel mei nata, Lauinia est, subdistinctione posita post natam; contra autem nomine quidem cognito, ignorata uero cuius esset nata, dixisset Lauinia, mea nata est, post Lauiniam subdistinguendo (GL 3, 213.18-25)

Virgile en VII: est mihi nata... [j'ai une fille, En. 7, 268], à la place de possideo natam. Cela étant, on emploie plutôt le datif quand on s'adresse à des gens qui en quelque sorte ne sont au courant de rien, comme dans le vers ci-dessus; si en revanche il s'était adressé à des gens qui auraient su qu'il avait une fille, mais qui auraient ignoré son nom, il aurait dit mea ou mei nata, Lauinia est [ma fille, c'est Lavinia], en mettant une pause après nata; et à l'inverse, si on avait connu son nom, tout en ignorant de qui elle était la fille, il aurait dit Lauinia, mea nata est (Lavinia, c'est ma fille), en mettant une pause après Lauinia. 
En soulignant que les variations de formulation sont fonction du type de connaissance que le locuteur et l'allocutaire ont de la situation, cet exemple montre à quel point cette problématique cognitive est essentielle dans la conception que Priscien se fait de l'articulation entre les domaine intra- et extra-linguistique.

La situation se complique en outre du fait que cette conception ne trouve pas sa clé dans l'emploi de tel ou tel terme particulier, mais dans le réseau que différents termes constituent les uns avec les autres: suppositum bien sûr, que l'on traduit usuellement par "référent", mais aussi substantia (qui forme couple avec qualitas), persona, agens et patiens. C'est ce réseau de termes qu'il convient d'examiner pour préciser comment s'organise chez Priscien le mécanisme cognitif de la construction et de l'identification de la référence.

Suppositum, calque du grec hupokeimenon, apparaît en bout de chaîne, comme le terme correspondant à l'élément le plus extérieur, en ce sens que ce qu'il désigne est entièrement indépendant de l'énoncé qui l'évoque et y renvoie ${ }^{12}$ : à cet égard, l'élément désigné par le terme suppositum est à proprement parler extra-linguistique. Comme le montre l'étymologie du terme grec calqué en latin, cet élément, "placé à la base" pourrait-on dire, est l'objet physiquement présent de la visée déictique, ce qu'on a sous les yeux.

Un passage peut paraître se prêter à une interprétation différente. En GL 3, 145.16-21, à propos de ce que signifient les noms, Priscien indique qu'ils ont été institués pour exprimer des caractéristiques (qualitates) communes ou propres, et il cite pour exemple homo et Plato, puis, en esquissant une opposition avec les pronoms, il remarque que cette visée sémantique des noms a entraîné la création d'une infinité de noms: "on en a institué un nombre infini, de ces noms, pour faire correspondre à chacun des référents la qualité qui lui est propre" (innumerabilis in eis, id est nominibus, positio fiebat, ut singulorum figuratio nominum singulis reddat suppositorum suam qualitatem). Dans une note de commentaire à propos du passage d'Apollonios dont Priscien s'est inspiré ici, Lallot (1997, 2: 90-91, note 38 ad Synt. $2,22)$ en tire une conclusion qui donne au suppositum un sens très large: "Par "référent" (...) il faut entendre ou bien un objet singulier, doué d'une qualité propre (idia poiotês), lorsqu'il s'agit d'un nom propre, ou bien une classe d'objets partageant une qualité commune (koinê poiotês) lorsqu'il s'agit d'un nom commun". En fait, au moins pour le texte de Priscien, il paraît plus vraisemblable que le suppositum est toujours ici le référent comme individu particulier, mais qu'il porte tantôt un nom

12 Sur le suppositum, cfr. Lallot (1997: 2, 26, note 94 du livre 1), qui critique l'interprétation, en effet trop large, que je proposais quelques années auparavant (1989: 392). 
qui exprime une caractéristique propre (qualitas propria: Plato), tantôt un nom qui exprime une caractéristique "commune", en ce qu'il la partage avec d'autres (qualitas communis: homo).

Ce terme suppositum est relayé dans certains passages du livre 17 de l'Ars par unusquisque [chacun] ou omnes [tous] (GL 3, 146.1-3), voire par omnis homo [tout homme] (GL 3, 149.27-28). Cette assimilation entre le suppositum et des termes qui correspondent spécifiquement à des animés n'implique pas forcément que le référent ne puisse être qu'un animé: cela paraît plutôt dû en fait à l'intérêt qui est porté dans le livre 17 à la définition du nom. Priscien souligne en effet que les supposita ont en eux-mêmes un ensemble de caractères, de propriétés, ce qu'il appelle leur qualitas (cf. GL 3, 145.16-146.4 et particulièrement 145.21) ; or dans la conception de Priscien cette qualitas des supposita est précisément ce qu'expriment les noms - avec cette particularité que les caractères communs à plusieurs référents relève des noms "appellatifs", cependant que les noms "propres" expriment des caractères qui appartiennent en propre à un référent (au moins en principe ${ }^{13}$ ). Cette structure sémantique invite à considérer prototypiquement comme référents les entités susceptibles de porter non seulement un nom commun, mais également un nom propre, et donc les animés... Mais cela n'interdit nullement de penser que les référents puissent être des non animés. Ainsi, dans les très scolaires Partitiones, Priscien donne une définition du nom où le suppositum apparaît sous la forme res supposita (GL 3, 464.33). Cette imprécision dans la présentation du suppositum (animé, inanimé, les deux?) tient encore une fois au fait que cette notion correspond à une entité extérieure au langage, et qui en tant que telle n'intéresse Priscien qu'au travers du mécanisme linguistique qui permet d'y accéder - mais rien de plus. Si en effet il est question, comme on va le voir, de la substantia suppositi (GL 3, 122.1-2 et 129.13-14) et de la qualitas suppositi, ou suppositorum (145.21 et 149.10), le suppositum lui-même n'est déterminé par rien et ne reçoit pas l'amorce d'une description. La seule question qui est posée à son sujet est de savoir s'il est connu du locuteur ou de l'allocutaire, et dans quelle mesure.

La substantia de son côté correspond à l'être considéré en lui-même, qu'il soit ou non caractérisé, c'est-à-dire qu'il soit accompagné ou non d'une qualitas. Les amples développements consacrés à l'opposition entre l'expression de la substantia seule ou l'expression concomitante de la substantia et de la qualitas permettent de distinguer d'un côté les pronoms, qui, n'exprimant que la substantia, peuvent faire

13 Sur les problèmes soulevés par les noms propres, cf. Groupe Ars Grammatica (2010: notes $47,64,88,102,103,169)$. 
référence à n'importe quel être, comme ego, tu, ille, et les noms, comme homo ou Plato, qui, exprimant à la fois une substantia et une qualitas, font référence à des êtres en tant qu'ils sont caractérisés - que ces caractères soient communs à une classe d'êtres (homo) ou qu'ils soient censés être propres à un être particulier (Plato). Ces deux modes de construction de la référence opposent ainsi le mode pronominal (un signe vide en langue est rempli en situation de discours par la deixis ou l'anaphore), et le mode nominal (un signe descriptif en langue sert à identifier un référent par ses caractéristiques, sans recours à la deixis) ${ }^{14}$.

Lorigine de cette notion de substantia est clairement aristotélicienne, comme pour le suppositum, et il est encore possible de distinguer chez Priscien, comme chez Apollonios, la trace de l'opposition entre substance première et substance seconde telle qu'elle apparaît dans les Catégories d'Aristote: de même que, dans les termes mêmes de ce dernier, la substance première, non qualifiée, "fait substrat pour tout le reste", et la substance seconde, comme "homme", "être animé", signifie "une certaine substance qualifiée" (Cat. 3b 21), cette opposition demeure dans la distinction établie par ces grammairiens entre l'expression de la seule substantia par le pronom, et l'expression à la fois de la substantia et de la qualitas par le nom. Cette trace n'est pas négligeable en elle-même, mais elle n'a en somme qu'un intérêt archéologique: les deux notions de substantia et de qualitas fonctionnent, dans ce contexte d'analyse linguistique, indépendamment du cadre où elles sont apparues, et leur interprétation dans le texte de Priscien ne nécessite pas d'être liée à leur origine ${ }^{15}$.

La question qui importe ici, c'est la portée de la notion de substantia dans le cadre de la problématique de la référence: qu'est-ce que la substantia par rapport à un référent, et plus particulièrement, quel est le rapport entre substantia et suppositum? À la différence de l'entité extra-linguistique qu'est le suppositum, la substantia, et la qualitas, c'est-à-dire l'"être" et les "propriétés" ou "caractéristiques" que cette entité partage avec d'autres ou possède en propre, ont pour fonction d'intégrer le référent dans le plan linguistique, c'est-à-dire de rendre compte du référent à l'intérieur du cadre linguistique, en en faisant le principe de la description sémantique du nom et du pronom: la substantia de Priscien, c'est l'être en tant qu'il est signifié par un substantif, nom, pronom, voire verbe "être" (appelé précisément uerbum substantiuum). En somme, contrairement au suppositum, entité extra-linguistique, la substantia correspond à cette entité, mais sur le plan sémantique, et en tant que telle

14 Sur cette opposition telle qu'elle est mise en place par Apollonios, cf. Lallot (1997, 2: note 31 du livre 2)

15 Sur la substantia chez Priscien, cf. Garcea (2009). 
elle signifie l'être non pas considéré en lui-même, tel qu'il est censé être présent dans le monde auquel le locuteur fait référence, comme c'est le cas du suppositum, mais l'être médiatisé par son expression, et donc en tant qu'il est intégré à une catégorie linguistique. Examinant ainsi une interrogation du type quis est Trypho?, Priscien écrit (GL 3, 129.12-14): (quis) quando nomini adiungitur, substantiam definitam in aliqua certa persona quaerimus suppositi; hanc enim solam ostendunt pronomina ("quand quis est associé à un nom, on cherche la substance déterminée qui se trouve dans une personne donnée, le référent: c'est en effet cette substance et elle seule que désignent les pronoms"). Cette formulation complexe montre que Priscien distingue nettement la substantia et le suppositum. L'objet de l'interrogation considérée (ce que "nous cherchons"), c'est assurément Tryphon, comme référent extra-linguistique, c'est-à-dire le suppositum, mais en tant que substantiam definitam, donc en tant qu'il est signifié par le pronom, qui sert ainsi de médiation pour y parvenir, et par opposition à ce que serait une interrogation sur telle ou telle des caractéristiques de cet être (ses qualitates), qui seraient signifiées par le nom. En d'autres termes, le suppositum correspond au référent en tant qu'il est indépendant de la langue, alors que la substantia et ses propriétés, ou caractéristiques, l'inscrivent dans sa dimension sémantique: pas de substantia en dehors de la langue, pas de substantia sans un nom, un pronom ou un verbe qui l'énonce, dans la mesure où elle correspond à de l'être en tant qu'il est signifié par le nom ou le pronom, voire par le verbe "être".

Comment s'insère la persona dans ce dispositif?

La complexité des sens de ce terme a été largement décrite ${ }^{16}$. Lorsqu'il apparaît chez Plaute, employé au pluriel, persona, qui désigne d'abord le masque, prend par métonymie le sens de "personnages" de théâtre, puis en vient à désigner les rôles ou les types comportementaux qu'illustrent les personnages littéraires. A partir de là, deux acceptions se développent, selon que le terme conserve son sens de "rôle", ou s'élargit pour correspondre plus généralement aux caractéristiques qui permettent de définir en propre une personne. Dans ce dernier sens, persona désigne l'ensemble des traits de caractère et des comportements qui constituent un individu - et finalement l'individu lui-même considéré globalement.

Des éléments de ces deux sens, "rôle" et "individu", qui n'ont rien de propre aux contextes grammaticaux, apparaissent dans l'Ars de Priscien, et s'entremêlent, ou du moins convergent. Persona y désigne en effet fréquemment l'individu en qui s'incarne un rôle, une fonction. Ce sens s'observe notamment dans les tournures

16 Cf. Nédoncelle (1948: 296-298), Moussy (2001: 158-161), Guérin (2009: 15-17). 
où persona est déterminé par un nom de fonction au génitif, du type persona agentis ("la personne de l'agent"), beaucoup plus fréquent que le rare persona agens ("la personne agissant"), quoique les contextes soient similaires: le génitif de définition, qui comporte l'indication du rôle occupé par l'individu concerné, paraît plus adapté au sens même de persona. Outre "l'agent et le patient" (personas afficientis et afficiendi, id est agentis et patientis, 163.26, et cf. 164.18), ce type de tournure se trouve avec "celui qui désigne et celui qui est désigné" (personis ostendentis et eius qui ostenditur 149.11-12), ainsi qu'avec "le possesseur et le possédé" (personas... possessoris et possessionis 165.15). Dans toutes ces tournures, persona est employé pour souligner que les activités citées constituent des rôles, des fonctions occupées par des individus spécifiques.

Par là-dessus, persona a dans le contexte des grammaires un sens particulier. Dans ce type de textes, ce terme est employé en effet pour l'essentiel dans un cadre énonciatif - comme c'était déjà le cas du terme grec prosôpon, dont persona est le calque latin ${ }^{17}$. Dans ce cadre, persona représente les trois composantes du processus énonciatif: le locuteur, l'allocutaire, et celui sur qui porte l'énoncé produit au cours de ce processus ${ }^{18}$. C'est à partir de là que la notion de persona, mettant sur le même plan les trois rôles constitutifs de ce processus, porteurs chacun de marques morphologiques spécifiques, a servi à désigner la catégorie grammaticale elle-même de la "personne" - ce qui est un sens fréquent de ce terme dans les contextes grammaticaux.

Dans ses emplois spécifiquement grammaticaux, persona peut donc représenter à la fois l'individu qui occupe l'un des rôles du processus énonciatif, et la catégorie grammaticale qui permet d'exprimer les oppositions correspondantes, c'est-à-dire les $1^{\text {re }}, 2^{\mathrm{e}}$ et $3^{\mathrm{e}}$ personnes. Accessoirement, la mise sur le même plan de ces trois rôles explique également que la " 3 e personne" soit prototypiquement considérée comme un animé - sur le modèle des deux premières.

La dimension énonciative qui détermine ces emplois proprement grammaticaux a pris dans ces textes une place primordiale. En effet, même lorsqu'il

17 Sur la place prépondérante de l'énonciation dans la construction du concept de personne, cf. Julien (1985), Lambert (1986), Lallot (1997, 2: notes 137, 139 et 363 du livre 3).

18 GL 2, 584.11-14: prima est cum ipsa quae loquitur de se pronuntiat; secunda cum de ea ad quam directo sermone loquitur; tertia cum de ea quae nec loquitur nec ad se directum accipit sermonem [on a la $1^{\text {re }}$ personne quand la personne même qui parle s'exprime à son propre sujet; la $2^{\mathrm{e}}$ quand elle s'exprime au sujet de celle à qui elle parle en lui adressant directement la parole; la $3^{\mathrm{e}}$ quand elle s'exprime au sujet de celle qui ni ne parle ni ne se fait adresser directement la parole]. Sur cette définition, cf. Amacker (1990: 271). 
ne s'agit pas explicitement des $1^{\text {re }}, 2^{\mathrm{e}}$ et $3^{\mathrm{e}}$ personnes, la persona, en raison de l'importance de cette tripartition, apparaît comme une entité susceptible d'entrer dans cette répartition des rôles énonciatifs, et s'entend ainsi fondamentalement par rapport à ce triptyque: toute personne est nécessairement le locuteur, l'allocutaire ou celui sur qui porte l'énoncé. En revanche, à l'intérieur de ce cadre énonciatif, la persona peut représenter indifféremment:

a) un individu de la réalité extralinguistique; lorsque par exemple Priscien écrit (118.19-20) "si dicam ego et tu intellegimus nihil addens, ad ceteras omnes personas discernitur" [si je dis ego et tu intellegimus [toi et moi nous comprenons], sans rien ajouter, c'est par opposition à toutes les autres personnes], il est clair que par rapport à ego et tu, la formule ad ceteras omnes personas discernitur ne peut viser la $3^{\mathrm{e}}$ personne comme catégorie, mais tous les individus qui ne sont ni le locuteur ni l'allocutaire;

b) la notion de cet individu; ainsi, lorsque Priscien évoque "la personne que l'on comprend dans le nom lui-même" (persona ea quae in nomine ipso intellegitur, 145.23-24), pour préciser qu'"elle est restée indéterminée" (caruit definitione), il ne s'agit pas de l'individu du domaine extralinguistique, mais de ce qui est signifié par son nom;

c) un actant de l'énoncé; la transitivité par exemple est décrite comme le "passage" (transitio) d'une personne à une autre (cf. 127.16-21; 148.1; etc) - et se distingue ainsi de la "réflexion" (reciprocatio, cf. 164.22-26) dans la mesure où les tournures réfléchies sont caractérisées par le retour de la même personne vers elle-même;

d) la personne comme catégorie grammaticale $\left(1^{\mathrm{re}}, 2^{\mathrm{e}}, 3^{\mathrm{e}}\right)$.

Cette élasticité induit dans de nombreux passages la superposition, dans la persona, de la personne-individu et de la personne-catégorie. Cette "confusion"19, déjà sensible chez Apollonios, est largement soulignée dans les analyses consacrées à la personne dans les textes grammaticaux anciens, qu'elle soit simplement constatée (Amacker 1990) ou fasse l'objet de tentatives d'explication, parfois très sophistiquées (Julien 1985, Lambert 1986). Les hypothèses et reconstructions avancées témoignent surtout de la gêne des Modernes devant une indistinction qui leur paraît d'autant

19 Cf. Amacker (1990: 272), "Priscien confond de manière inextricable les personnes grammaticales et les personnes physiques". 
plus insupportable que ceux qui la commettaient semblent avoir eu tous les moyens de l'éviter.

Cette élasticité se retrouve à un autre niveau. Comme déjà chez Apollonios, où la personne peut-être un cheval ou une maison (Lallot, 1997: II, n. 12), l'extension de la persona dépasse à l'occasion la personne humaine: au début du livre 18 (212.2224), pour illustrer un changement de personne (ad alias transeunt uerba personas), Priscien cite quatre exemples, dont l'un comporte Hector, mais les trois autres uictoria, Troia, Graecia. Ce n'est bien sûr pas toujours le cas, et on a pu légitimement insister sur l'attachement de Priscien à la distinction entre animés et inanimés ${ }^{20}$, mais s'agissant de la persona, le processus de désémantisation est clairement entamé.

En fait, la dimension énonciative caractéristique de la notion de personne lui confère surtout une valeur d'opposition, de contraste, qui est sous-jacente à la plupart de ses emplois: une persona est une entité dont la caractéristique propre est de s'opposer à d'autres. Cette opposition est sans doute à l'origine même de la notion de transitivité, si celle-ci provient bien de la perception d'une opposition d'actants, décrite en termes de transfert d'un actant à un autre.

Comment s'articulent la persona et la substantia dans le texte de Priscien?Le premier passage où ces deux notions sont combinées, au début du livre 17 , fait entrevoir une première difficulté:

Nomina enim tertiarum sunt personarum indicatiua, id est tertias indicant personas, quippe quae nomina substantiam et qualitatem uel generalem uel propriam significantia similiter in omnibus possunt intellegi personis inesse. Omnis enim persona et generalis et specialis capax est substantiae et qualitatis; potest enim homo esse, qui loquitur et ad quem loquitur et de quo aliquis loquitur, et Cicero dici, qui loquitur et ad quem loquitur et de quo aliquis loquitur. Itaque quia confusio infinitam faciebat personam, concessit ad sibi aptam, id est tertiam (GL 3, 117.11-19)

Les noms en effet sont indicatifs de tierces personnes (c'est-à-dire qu'ils indiquent des troisièmes personnes). Dans la mesure où ils signifient la substance et sa qualité, qu'elles soient génériques ou propres, on peut les comprendre comme inclus dans toute personne indistinctement: toute personne en effet est le réceptacle d'une substance et de sa qualité, qu'elles soient génériques ou spécifiques

20 Colombat (2009: 300-305) en donne des exemples convaincants. 
(peut être homme en effet celui qui parle, celui à qui il parle, et celui dont on parle, tout comme peut être appelé Cicéron celui qui parle, celui à qui il parle, et celui dont on parle). Une telle ambiguité provoquait l'indétermination de la personne, et a abouti à celle qui était adaptée à cette ambiguïté même, c'est-à-dire la troisième.

Ce passage peut paraître tout à fait ambigu, et c'est ce genre d'ambiguïté qui a conduit certains Modernes à parler de confusion. La personne étant en effet présentée dans ce passage comme étant qui loquitur et ad quem loquitur et de quo aliquis loquitur, et susceptible de porter les noms d'homme et de Cicéron, il n'est pas illégitime de comprendre que persona représente ici un individu particulier, appartenant à la réalité extra-linguistique, et considéré dans le cadre du triptyque énonciatif: toute personne concrète, qu'elle occupe le statut de locuteur, d'allocutaire ou de tierce personne, est susceptible de porter un nom appellatif (homme) ou propre (Cicéron). Mais la formule capax substantiae, "réceptacle d'une substance", qui définit ici la persona, modifie l'interprétation. Il serait douteux que Priscien ait voulu dire que tout individu de la réalité extra-linguistique peut être le "réceptacle d'une substance": cela parait trop évident; persona doit plutôt être compris comme représentant un rôle, une place du dispositif énonciatif, place destinée à être occupée par de la substantia, c'est-à-dire par de l'être, et en tant que tel correspondre à un individu porteur d'un nom. Il semble donc y avoir ici au moins une ambiguïté dans le terme persona, entre l'individu, entité extra-linguistique, et la place dans le dispositif énonciatif.

En réalité, cette ambiguité est plus apparente que réelle. Le fond de l'affaire, c'est que la persona, qui représente bien le locuteur, l'allocutaire ou une tierce personne, n'est pas une substantia: elle représente des individus sous l'angle pragmatique de leur fonction dans le dispositif énonciatif, et non sous l'angle sémantique de l'être signifié par leur nom. Ce dont persona se rapproche le plus, ce n'est pas de substantia, mais de suppositum. La dimension énonciative de persona fonctionne en effet indifféremment dans le plan intra- et extralinguistique, et par conséquent il peut y avoir à l'occasion coïncidence des deux perspectives, et assimilation du rôle énonciatif tel qu'il est catégorisé dans la grammaire et de la personne concrète qui l'occupe dans le domaine extralinguistique.

Par exemple en 151.2-4, à nouveau à propos du passage de Virgile (Én. 6, 789) où Anchise aux Enfers dit à Énée "hic Caesar" en lui montrant César: ut autem qualitatem quoque propriam ostenderet personae quam demonstraret, addidit Caesar [pour indiquer ce qui caractérise en propre la personne qu'il désignait, il ajouta Caesar]; la persona désignée ici (personae quam demonstraret) est une entité du domaine 
extra-linguistique, c'est-à-dire à proprement parler un suppositum, même si cela se passe dans un monde fictif. Cela étant, c'est un contexte particulier qui détermine cette synonymie: dans tout ce passage du livre $17 \mathrm{il}$ s'agit de la $3^{\mathrm{e}}$ personne, de la relation des noms à cette $3^{\mathrm{e}}$ personne, et du rôle des pronoms de cette même personne; l'exemple de Virgile a pour fonction d'illustrer l'emploi concomitant d'un pronom déictique et d'un nom, tous deux à la $3^{\mathrm{e}}$ personne, ce qui a apparemment contribué ici à la généralisation de l'emploi de persona pour désigner à la fois une catégorie linguistique, la $3^{e}$ personne, et le référent auquel en l'occurrence ce nom à la $3^{\mathrm{e}}$ personne correspond.

Le second passage du livre 17 où ces deux notions sont organisées l'une par rapport à l'autre peut paraître quant à lui en contradiction avec le précédent:

Et certum quod accidentium est eius significationi, id est substantiae, quae innascitur persona (GL 3, 140.23-24)

Il est évident que ce que signifie le pronom, à savoir la substance, a parmi ses accidents ce qui émane de celle-ci, c'est-à-dire la personne.

Ici, dans la relation entre persona et substantia, la substance est première: la personne en est l'émanation, elle en "naît" (innascitur) - ce qui surprend par rapport au passage précédent, où la persona était définie comme capax substantiae, "réceptacle d'une substance".

Dans les deux passages, la substantia correspond à de l'être dans la mesure où il s'inscrit dans le cadre linguistique, en tant qu'il est signifié par le nom et le pronom; quant à la persona, elle renvoie tout autant au processus énonciatif dans ce second passage que dans le premier: être le locuteur, l'allocutaire ou une tierce personne est une caractéristique de la substance exprimée par un nom ou un pronom, et c'est à ce titre que la personne est un accident de la substance. Ce qui change d'un texte à l'autre, c'est le point de vue: à partir de la substantia, la persona est seconde dans la mesure où une perspective élémentairement ontologique suppose que l'être lui-même est antérieur à sa répartition dans le dispositif énonciatif, comme locuteur, allocutaire ou tierce personne; mais si la relation entre les deux notions est considérée à partir de la persona, cette dernière apparaît comme le réceptacle dans lequel la substantia prend forme et s'incarne.

En revanche, encore une fois, le fait que la persona représente la personne grammaticale ou la personne physique est une distinction secondaire à ce niveau, voire inopérante. La persona constitue plutôt une valeur d'opposition, appliquée 
indifféremment à une entité extra-linguistique, distincte de toutes les autres, ou à une catégorie grammaticale, dans la mesure où chacune des trois positions énonciatives concernées a pour caractéristique première de se distinguer des deux autres.

La combinaison des différentes notions examinées ici apparaît dans de rares passages du livre 17, et notamment en 129.13-14, à propos de l'interrogation quis est Trypho?. Le commentaire de Priscien, déjà évoqué, regroupe les termes substantia, persona et suppositum: substantiam definitam in aliqua certa persona quaerimus suppositi. Lobjet de la question quis est Trypho?, pour laquelle il est précisé que la réponse peut être ego, ou tu, ou ille [moi / toi / lui], porte sur l'identification au niveau sémantique d'un être particulier (substantiam definitam), en tant qu'il est pragmatiquement intégré au triptyque énonciatif (in aliqua certa persona) et correspond à un référent extralinguistique (suppositi). En termes plus contemporains, on pourrait dire qu'il s'agit de "chercher à situer dans le dispositif énonciatif l'être sémantiquement identifiable qui correspond à une entité extralinguistique".

Deux derniers termes viennent enrichir et compléter le système formé par la combinaison de suppositum, substantia et persona. Il s'agit d'agens et de patiens, qui s'entendent par rapport à la diathèse verbale, active ou passive, et permettent de rendre compte du cas pris dans l'énoncé par le nom ou le pronom, donc sur un plan syntaxique. Un passage rapproche tout particulièrement ces différentes notions, à l'exception du suppositum:

recta ordinatio exigit ut pronomen uel nomen praeponatur uerbo, ut ego et tu legimus, Virgilius et Cicero scripserunt, quippe cum substantia et persona ipsius agentis uel patientis, quae per pronomen uel nomen significatur, prior esse debet naturaliter quam ipse actus, qui accidens est substantiae (GL 3, 164.16-20)

la correction de l'agencement demande que pronom ou nom soient pré-posés au verbe, comme ego et tu legimus [toi et moi nous lisons], Vergilius et Cicero scripserunt [Virgile et Cicéron ont écrit], dans la mesure où la substance, et plus précisément la personne de l'agent lui-même, ou celle du patient, c'est-à-dire ce que signifie le pronom ou le nom, doit naturellement précéder l'acte lui-même, qui n'est qu'un accident de la substance.

Ce passage comporte un intéressant effet de progression avec la formule substantia et persona ipsius agentis uel patientis: 1'"être" se précise dans la "personne", et celle-ci est déterminée sous ses deux faces d'“agent" ou de "patient". 
Plutôt donc qu'une notion centrale, comme celle de référent, on trouve chez Priscien un ensemble complexe. Construit sur des critères distincts, cet ensemble permet d'aborder la question de la construction et de l'identification de la référence à plusieurs niveaux: dans le domaine qu'on pourrait dire proprement référentiel, c'est-à-dire extra-linguistique, réel ou fictif, avec le suppositum, entité du monde connu du locuteur et/ou de l'allocutaire; dans le cadre sémantique d'une catégorie linguistique déterminée (nom, pronom, verbe "être"), avec la substantia, "être" en tant qu'il est inscrit dans le domaine linguistique; sur un plan pragmatique dans le processus énonciatif avec la persona, protagoniste jouant un rôle particulier qui le distingue des autres; enfin d'un point de vue syntaxique par rapport au procès luimême, qu'il réalise ou subit, avec l'agens et le patiens, actant d'un procès exprimé par le verbe. L'une des difficultés du système ainsi mis en place tient, comme souvent dans le vocabulaire technique antique, à la porosité des frontières entre ces notions, qui se chevauchent à l'occasion.

Ce que cerne cet ensemble de notions, c'est ce qu'on appelle aujourd'hui un acteur, au sens où l'on parle des acteurs d'une négociation, c'est-à-dire un être, réel ou imaginaire, qui se définit par le rôle qu'il joue dans un événement. Cet acteur a pour prototype un animé, un être humain, mais rien n'interdit qu'à l'occasion, dans telle ou telle situation ou contexte particulier, ce soit autre chose. Lessentiel est que cet acteur, à travers les notions qui le cernent, s'entende toujours par rapport à l'énoncé où il figure. Alors que la notion contemporaine de "référent" suggère un mouvement centrifuge, de l'énoncé vers l'extérieur de celui-ci, dans le domaine appelé précisément "extra-linguistique", la notion latine de sup-positum implique plutôt l'idée d'un soubassement, comme celle de sub-stantia, et les autres, persona, agens / patiens, celle de rôles. Ensemble, elles dessinent l'image d'un jeu d'éléments qui sont pensés par rapport à l'énoncé - en quelque sorte dans un mouvement centripète.

\section{RÉFÉRENCES BIBLIOGRAPHIQUES}

Amacker René (1990), "L’argumentation pragmatique chez Priscien: 'personne’ et 'déixis”', dans Historiographia Linguistica XVII 3, p. 269-291.

Apollonios, Syntaxe: voir Lallot (1997).

BARATin Marc (1989), La naissance de la syntaxe à Rome, Paris, Éditions de Minuit. Colombat Bernard (2009), "Les fondements sémantiques de la transitivité: à la recherche de la personne”, dans Pierrard M. et van Raemdonck D. (éd.) 
Représentation du sens linguistique III (Bruxelles, 3-5 novembre 2005), Gembloux, Duculot.

Garcea Alessandro (2009), "Substance et accidents dans la grammaire de Priscien", dans Baratin M., Colombat B., Holtz L. (éd.), Priscien: transmission et refondation de la grammaire, de l'Antiquité aux Modernes, Turnhout, Brepols (Studia Artistarum, 21), p. 125-138.

Grammatici Latini [GL], ed. Keil Heinrich, 1-7, Leipzig, Teubner, 1855-1880 (réimpr. Hildesheim / New York, Olms, 1961).

Groupe Ars Grammatica (Baratin Marc et al.) éd. (2010), Priscien, Grammaire, livre XVII, Syntaxe, 1. Texte latin, traduction introduite et annotée. Paris,Vrin.

GuÉRIN Charles (2009), Persona. L'élaboration d'une notion rhétorique au Ier siècle av. J.-C., vol. I, Paris, Vrin.

KLEIBER Georges (sans date: 1996 ou 1997), "Référents évolutifs et pronoms: une suite", dans Recherches linguistiques 20, p. 115-148.

Kleiber Georges (1999), Problèmes de sémantique. La polysémie en questions, Lille, Presses universitaires du Septentrion.

Lallot Jean (1997), Apollonius Dyscole De la Construction, Vol. 1: Introduction, texte et traduction; Vol. 2: Notes et index, Paris, Vrin.

Lambert Frédéric (1986), "Aspects de l'énonciation chez Apollonius Dyscole", dans HEL VIII-2, p. 39-52.

Moussy Claude (2001), "Esquisse de l'histoire du substantif persona", dans Alvar Ezquerra A. et Garcia Jurado F. éd., Actas del X congreso español de estudios clásicos, vol. 2, Madrid, Sociedad Española de Estudios Clásicos, p. 153-161.

NéDONCElle Maurice (1948), "Prosopon et persona dans l'antiquité classique", dans Revue des sciences religieuses, 22, p. 277-299.

Priscien, Ars grammatica, livre 17: voir Groupe Ars grammatica (2010).

Baratin, M. Acerca do referente, uma construção original da lingüística antiga tardia.

RESUMO: $O$ artigo propõe-se reconstituir o sistema conceitual em que se desenvolve a noção de persona na Ars grammatica de Prisciano. Mais que uma noção central, porém, o que lá se encontra é um sistema 
complexo que, construído segundo critérios distintos, permite abordar a questão da construção e da identificação da referência em diversos níveis: a) no plano propriamente referencial, isto é, extralingüístico, real e fictício, com o suppositum, entidade do mundo conhecido do locutor e/ou alocutário; b) no plano semântico de uma categoria lingüística determinada (nome, pronome, verbo "ser"), com a substantia, "ser" na medida em que se insere no domínio lingüístico; c) no plano pragmático do processo enunciativo, com a persona, protagonista que desempenha um papel particular que o distingue dos outros; d) no plano sintático relativo ao processo mesmo que ele realiza ou sofre, com o agens e o patiens, actante de um processo expresso pelo verbo. Uma das dificuldades de tal sistema deve-se, como freqüentemente no vocabulário técnico antigo, à porosidade das fronteiras entre tais noções, que às vezes se sobrepõem umas às outras.

PALAVRAS-CHAVE: Prisciano; referente; persona; suppositum e hypokeímenon; substantia e qualitas. 